University of St. Thomas, Minnesota

UST Research Online

$1-1-2014$

\title{
Conditions for a totally positive completion in the case of a symmetrically placed cycle
}

Charles R. Johnson

Brenda K. Kroschel

University of St. Thomas, Minnesota, bkkroschel@stthomas.edu

Follow this and additional works at: https://ir.stthomas.edu/cas_mathpub

Part of the Mathematics Commons

This Article is brought to you for free and open access by the Mathematics at UST Research Online. It has been accepted for inclusion in Mathematics Faculty Publications \& Data by an authorized administrator of UST Research Online. For more information, please contact asle4660@stthomas.edu. 


\title{
CONDITIONS FOR A TOTALLY POSITIVE COMPLETION IN THE CASE OF A SYMMETRICALLY PLACED CYCLE*
}

\author{
CHARLES R. JOHNSON ${ }^{\dagger}$ AND BRENDA K. KROSCHEL ${ }^{\ddagger}$
}

\begin{abstract}
In earlier work, the labelled graphs $G$ for which every combinatorially symmetric totally nonnegative matrix, the graph of whose specified entries is $G$, has a totally nonnegative completion were identified. For other graphs, additional conditions on the specified data must hold. Here, necessary and sufficient conditions on the specified data, when $G$ is a cycle, are given for both the totally nonnegative and the totally positive completion problems.
\end{abstract}

Key words. Totally nonnegative matrices, Totally positive matrices, Partial matrix, Matrix completion problem, Cycles.

AMS subject classifications. 15A48, 15A37.

1. Introduction. A matrix is totally positive (nonnegative) if all of its minors, principal or otherwise, are positive (respectively, nonnegative). Totally positive (TP) matrices and totally nonnegative (TN) matrices arise in a variety of applications including splines, statistics, and dynamical systems. A partial matrix is a rectangular array, in which some entries are specified while the remainder are free to be chosen from a relevant set of possibilities. A completion of a partial matrix is a matrix that results from some choice for the specified entries. A matrix completion problem asks when is there a completion of a desired type? A partial matrix is called partial TN (partial TP) if each of its fully specified minors is nonnegative (positive). Matrices that are TN satisfy the inheritance property in that every submatrix of a TN matrix is $\mathrm{TN}$ which gives the necessary condition that every fully specified submatrix of a partial TN matrix must be TN in order for there to be a TN completion. Matrices that are TP also satisfy the inheritance property.

For an $n$-by- $n$ matrix $A$ and index sets $\alpha, \beta \subseteq\{1,2, \ldots, n\}$, let $A[\alpha \mid \beta]$ be the submatrix of $A$ lying in the rows indexed by $\alpha$ and the columns indexed by $\beta$. For brevity let $A[\alpha]$ denote the principal submatrix of $A$ whose rows and columns are both indexed by $\alpha$.

${ }^{*}$ Received by the editors on August 11, 2009. Accepted for publication on October 19, 2010. Handling Editor: Harm Bart.

${ }^{\dagger}$ Department of Mathematics, College of William and Mary, Williamsburg, VA 23187-8795, USA (crjohnso@math.wm.edu).

${ }^{\ddagger}$ Department of Mathematics, Mail \#OSS 201, University of St. Thomas, 2115 Summit Avenue, St. Paul, MN 55105, USA (bkkroschel@stthomas.edu). 
The pattern of specified/unspecified entries in a partial matrix can be described by a graph. For an $n$-by- $n$ partial matrix $A$, the usual graph is $G=(V, E)$ with $V=N=\{1,2, \ldots, n\}$ in which the edge $\{i, j\}=i j$ is in $E$ if and only if $a_{i j}$ is specified. Only combinatorially symmetric partial matrices, that is matrices in which $a_{j i}$ is specified if and only if $a_{i j}$ is specified, are considered here. We also assume that the diagonal entries are specified and nonzero. As a result we consider only undirected graphs with loops omitted. A (simple) cycle is a sequence of vertices $v_{i}, v_{i+1}, \ldots, v_{k}, v_{i}$ for which $v_{j} v_{j+1} \in E$ and $v_{j} \neq v_{t}$ for $j \neq t$, and $j, t=i, i+1, \ldots, k$. Here, we are interested in both the TN and the TP completion problems in the case in which the specified entries form a symmetrically placed cycle and the diagonal entries are specified and positive. An analogous situation in the positive definite case has been studied in [1] and [2]. In the positive definite case, and this case as well, there must be conditions, in addition to partial positive definiteness ( $\mathrm{TP}$ or $\mathrm{TN}$ ), on the data. These obvious conditions, of course, are inherited by (partial) principal submatrices.

The graphs for which the inheritance property is sufficient to ensure the TN (TP) completion of a partial TN (TP) matrix were identified in [8] in the TN case and [9] in the TP case. Necessary additional conditions on the data for a number of patterns not included in [8] and [9] are developed in [4]. As cycles are not among any of these patterns, different conditions on the data are necessary to obtain a TN (or TP) completion in the case of a cycle. Since TN matrices are not permutation similarity invariant, the labeling of the cycle is important, unlike the positive definite case. Here we concentrate upon the most natural labeling, in which the $i, j$ entry is specified when $j=i-1, i$ or, $i+1(\bmod n), i=1,2, \ldots, n$. The general case of an arbitrarily labeled cycle is more difficult, and is an open question both for TN and TP completions. Since the diagonal entries are positive and the TN matrices are invariant under multiplication by a diagonal matrix with positive diagonal entries, we may suppose that our partial matrix appears as

$$
A=\left[\begin{array}{ccccc}
1 & s_{1} & & & t_{n} \\
t_{1} & 1 & s_{2} & ? & \\
& t_{2} & \ddots & \ddots & \\
& ? & \ddots & \ddots & s_{n-1} \\
s_{n} & & & t_{n-1} & 1
\end{array}\right] .
$$

Note that TP matrices are TN and the above comments apply in the TP case as well.

We first develop necessary and sufficient conditions for a TN completion on a cycle with natural labelling in Section 2. In Section 3, we develop conditions for a TP completion in the case of a cycle with natural labelling. In this case, extra care must be taken since many minors of the completion in the TN case are zero and this must be avoided when finding a TP completion. This requires use of a different completion 
strategy, although the idea of building the completion by induction is similar to that of the TN case. Some closing observations are made in Section 4.

2. The totally nonnegative cycle completion. We note that the initial observations of this section were also made in [10], along different lines, and in [3] (where in particular, the interesting completion (2.3) is displayed and its implications given).

We first derive the necessity of the additional conditions for $A$ to have a TN completion. Of course, $A$ is partial $\mathrm{TN}$ if and only if $s_{1}, \ldots, s_{n} \geq 0, t_{1}, \ldots, t_{n} \geq 0$ and $s_{i} t_{i} \leq 1, i=1, \ldots, n$, which we assume throughout. Consider now an $n$-by- $n$ TN matrix $B=\left(b_{i j}\right)$, with $b_{i i}=1, i=1, \ldots, n$. Assume, for the moment, that $b_{23}, \ldots, b_{n-2, n-1}>0$. We show, by induction on $n$, that

$$
b_{1 n} \leq b_{12} b_{23} \cdots b_{n-1, n}
$$

(and note that only the nonnegativity of 2 -by- 2 minors is used in the proof). In case $n=3$, this is just the statement that the upper right 2 -by-2 minor is nonnegative. Now, suppose that $n \geq 4$ and that we have the above statement valid when $n$ is replaced by any smaller value. In particular, we have

$$
b_{2 n} \leq b_{23} b_{34} \cdots b_{n-1, n}
$$

because the lower $(n-1)$-by- $(n-1)$ submatrix is $\mathrm{TN}$, and

$$
b_{13} \leq b_{12} b_{23},
$$

as the upper left 3 -by-3 submatrix is TN. But, since $B$ is TN, the 2 -by-2 submatrix

$$
\left[\begin{array}{ll}
b_{13} & b_{1 n} \\
b_{23} & b_{2 n}
\end{array}\right]
$$

has nonnegative determinant. Thus,

$$
b_{1 n} b_{23} \leq b_{13} b_{2 n} \leq b_{12} b_{23}^{2} b_{34} \cdots b_{n-1, n} .
$$

For $b_{23}>0$, this implies the claimed inequality. If $B$ is TN with positive diagonal, then, for any $j>i$, if $b_{i j}=0$ all entries above and/or to the right of $b_{i j}$ are also 0 . Thus, if our temporary assumption about positivity of super-diagonal entries is false, the desired inequality is still valid, as $b_{1 n}=0$.

We conclude that, in order for $A$ to have a TN completion, the additional conditions

$$
t_{n} \leq s_{1} s_{2} \cdots s_{n-1}
$$

and (by transposition)

$$
s_{n} \leq t_{1} t_{2} \cdots t_{n-1}
$$


are necessary.

We next verify the sufficiency of the above conditions, also by induction, beginning with $n=4$. When $n=4$ our partial TN matrix may be written as

$$
B=\left[\begin{array}{cccc}
1 & s_{1} & ? & t_{4} \\
t_{1} & 1 & s_{2} & ? \\
? & t_{2} & 1 & s_{3} \\
s_{4} & ? & t_{3} & 1
\end{array}\right]
$$

If $s_{1}, t_{3} \neq 0$, then one completion that is $\mathrm{TN}$ is the following:

$$
\hat{B}=\left[\begin{array}{cccc}
1 & s_{1} & s_{1} s_{2} & t_{4} \\
t_{1} & 1 & s_{2} & \underline{t_{4}} \\
s_{4} \\
t_{3} & t_{2} & 1 & s_{3} \\
s_{4} & t_{2} t_{3} & t_{3} & 1
\end{array}\right]
$$

The fact that this matrix is TN, given that the data meets the necessary conditions, may be verified directly. For example, the $\{2,3\},\{1,2\}$ minor is nonnegative because $s_{4} \leq t_{1} t_{2} t_{3}$. It is interesting that, even if the data were symmetric, this completion would not (and could not) be. A symmetric partial TN matrix, whose graph is a cycle, need not have a symmetric TN completion, even when the conditions for a TN completion are met.

If either $s_{1}$ (or $t_{3}$ ) is zero, then the entries in row one (row four) to the right of $s_{1}$ (to the left of $t_{3}$ ) must also be zero (see [5]). To complete the rest of the matrix, transpose and use the completion strategy above.

For the induction step in the proof that the conditions are sufficient for completion in the $n$-by- $n$ case, we partition the partial matrix meeting the necessary conditions into two overlapping blocks: the upper left 2 -by-2 and the lower right $(n-1)$-by- $(n-1)$. Given a completion of the lower right, we then try to arrange that the entries outside these blocks be what the monotonically labelled block-clique completion would have been for these two blocks [8], i.e., the $1, j$ entry should equal the 1,2 entry times the $2, j$ entry for $j \geq 3$, and similarly for the first column. If this can be arranged, we know from [8] that the result is TN (if the lower right block is). Choose the 2,n entry as $\frac{t_{n}}{s_{1}}$, and the $n, 2$ entry as $\frac{s_{n}}{t_{1}}$. Then the lower right block meets the necessary conditions and, thus, has a TN completion by induction. Now, note that in the $1, n$ position $t_{n}=s_{1}\left(\frac{t_{n}}{s_{1}}\right)$, and in the $n, 1$ position $s_{n}=t_{1}\left(\frac{s_{n}}{t_{1}}\right)$. No other entries, after the second, in the first row or column are specified. Thus, the above program may be carried out. For example, to complete in case $n=5$, given the completion for $n=4$, 
the sequence of steps is as follows:
$(1)\left[\begin{array}{ccccc}1 & s_{1} & ? & ? & t_{5} \\ t_{1} & 1 & s_{2} & ? & ? \\ ? & t_{2} & 1 & s_{3} & ? \\ ? & ? & t_{3} & 1 & s_{4} \\ s_{5} & ? & ? & t_{4} & 1\end{array}\right]$
$(2)\left[\begin{array}{ccccc}1 & s_{1} & ? & ? & t_{5} \\ t_{1} & 1 & s_{2} & ? & \frac{t_{5}}{s_{1}} \\ ? & t_{2} & 1 & s_{3} & ? \\ ? & ? & t_{3} & 1 & s_{4} \\ s_{5} & \frac{s_{5}}{t_{1}} & ? & t_{4} & 1\end{array}\right]$
$(3)\left[\begin{array}{ccccc}1 & s_{1} & ? & ? & t_{5} \\ t_{1} & 1 & s_{2} & s_{2} s_{3} & \frac{t_{5}}{s_{1}} \\ ? & t_{2} & 1 & s_{3} & \frac{t_{5}}{s_{1} s_{2}} \\ ? & \frac{s_{5}}{t_{1} t_{4}} & t_{3} & 1 & s_{4} \\ s_{5} & \frac{s_{5}}{t_{1}} & t_{3} t_{4} & t_{4} & 1\end{array}\right]$,

$\left[\begin{array}{ccccc}1 & s_{1} & s_{1} s_{2} & s_{1} s_{2} s_{3} & t_{5} \\ t_{1} & 1 & s_{2} & s_{2} s_{3} & \frac{t_{5}}{s_{1}} \\ t_{1} t_{2} & t_{2} & 1 & s_{3} & \frac{t_{5}}{s_{1} s_{2}} \\ \frac{s_{5}}{t_{4}} & \frac{s_{5}}{t_{1} t_{4}} & t_{3} & 1 & s_{4} \\ s_{5} & \frac{s_{5}}{t_{1}} & t_{3} t_{4} & t_{4} & 1\end{array}\right]$

This completes the proof and the general theorem is:

THEOREM 2.1. Suppose that

$$
A=\left[\begin{array}{ccccc}
a_{11} & a_{12} & & & a_{1 n} \\
a_{21} & a_{22} & a_{23} & ? & \\
& a_{32} & \ddots & \ddots & \\
& ? & \ddots & \ddots & a_{n-1, n} \\
a_{n 1} & & & a_{n, n-1} & a_{n n}
\end{array}\right]
$$

is a partial TN matrix with positive diagonal entries. Then A has a TN completion if and only if:

$$
a_{1 n} a_{22} \cdots a_{n-1, n-1} \leq a_{12} a_{23} \cdots a_{n-1, n}
$$

and

$$
a_{n 1} a_{22} \cdots a_{n-1, n-1} \leq a_{21} a_{32} \cdots a_{n, n-1} .
$$

Proof. Left multiply $A$ by $\operatorname{diag}\left(1 / a_{11}, \ldots, 1 / a_{n n}\right)$ and apply the above discussion.

Note that the entries $a_{11}$ and $a_{n n}$ are unconstrained by (2.4) and (2.5) and if either of these entries is unspecified they may be chosen as large as necessary to get a partial TN matrix of the form in Theorem 2.1. However, if an interior diagonal is unspecified, the values must be chosen to satisfy (2.4) and (2.5). 
3. The totally positive cycle completion. The completion used in [8] and the completion used here for the symmetrically placed cycle both have several minors that are zero. These completions are inherently TN and, although the same completions can be used to complete a partial TP matrix, the resulting completion would then be $\mathrm{TN}$, not TP. As a result, a TP completion is more difficult to achieve.

In the case of a partial TP matrix, the graph of whose specified entries is a symmetrically placed cycle, as given in (1.1), the necessary conditions for a TP completion are the same as in (2.1) and (2.2) with strict inequalities. That is,

$$
t_{n}<s_{1} s_{2} \cdots s_{n-1}
$$

and

$$
s_{n}<t_{1} t_{2} \cdots t_{n-1}
$$

As in the TN case, we begin by finding a TP completion in the case of a partial TP matrix, the graph of whose specified entries is a 4-cycle. To find a TP completion, begin by using the same completion as in the $\mathrm{TN}$ case and scale the completed entries pushing the minors away from zero as in Lemma 3.1.

LEMMA 3.1. Let

$$
A=\left[\begin{array}{cccc}
1 & s_{1} & ? & t_{4} \\
t_{1} & 1 & s_{2} & ? \\
? & t_{2} & 1 & s_{3} \\
s_{4} & ? & t_{3} & 1
\end{array}\right]
$$

be partial TP. Then the matrix

$$
\hat{A}=\left[\begin{array}{cccc}
1 & s_{1} & s_{1} s_{2} \frac{1}{r} & t_{4} \\
t_{1} & 1 & s_{2} & \frac{t_{4}}{s_{1}} \frac{s}{r} \\
\frac{s_{4}}{t_{3}} \frac{q}{p} & t_{2} & 1 & s_{3} \\
s_{4} & t_{2} t_{3} \frac{1}{p} & t_{3} & 1
\end{array}\right]
$$

with $p, q, r, s>1, t_{4}<s_{1} s_{2} s_{3}, s_{4}<t_{1} t_{2} t_{3}$

$$
r^{2}<s<r^{2}+\left(\alpha r-r^{2}\right) \frac{r-1}{r-s_{2} t_{2}}
$$

and

$$
p^{2}<q<p^{2}+\left(\beta p-p^{2}\right) \frac{p-1}{p-s_{2} t_{2}}
$$

for $\alpha=\frac{s_{1} s_{2} s_{3}}{t_{4}}$ and $\beta=\frac{t_{1} t_{2} t_{3}}{s_{4}}$ is $T P$. 
Proof. The choices for $p, q, r, s$ ensure that all necessary 2-by-2 minors are positive. In order to check that a matrix is totally positive, one only needs to check minors with consecutive rows and columns, and, in fact, by [6] only the initial minors need to be checked. The given inequalities (along with TP conditions on specified data) insure that all 2-by-2 minors as well as initial minors $\operatorname{det}(\hat{A}[1,2,3 \mid 2,3,4])$ and $\operatorname{det}(\hat{A}[2,3,4 \mid 1,2,3])$ are positive.

The first inequality in $(3.1)$ is from the $\{1,2 \mid 3,4\}$ minor. To get the second inequality in (3.1) note that

$$
\operatorname{det}(\hat{A}[1,2,3 \mid 2,3,4])=s_{1}\left(1-\frac{1}{r}\right)\left(s_{2} s_{3}-\frac{t_{4}}{s_{1}} \frac{s}{r}\right)-t_{4}\left(1-s_{2} t_{2}\right)\left(\frac{s}{r^{2}}-1\right) .
$$

This minor is positive if

$$
\left(1-s_{2} t_{2}\right)\left(s-r^{2}\right)<(r-1)(\alpha r-s) .
$$

Rearranging terms gives

$$
\begin{aligned}
s\left(r-s_{2} t_{2}\right) & <\alpha r(r-1)+r^{2}\left(1-s_{2} t_{2}\right) \\
& =\alpha r(r-1)-r^{2}(r-1)+r^{2}\left(r-s_{2} t_{2}\right) \\
& =\left(\alpha r-r^{2}\right)(r-1)+r^{2}\left(r-s_{2} t_{2}\right)
\end{aligned}
$$

or

$$
s<\left(\alpha r-r^{2}\right) \frac{r-1}{r-s_{2} t_{2}}+r^{2} .
$$

From positivity of the 2 -by-2 minors we also have $s<\alpha r$, but $1>s_{2} t_{2}$ gives $\frac{r-1}{r-s_{2} t_{2}}<1$ so that

$$
\left(\alpha r-r^{2}\right) \frac{r-1}{r-s_{2} t_{2}}+r^{2}<\alpha r
$$

and the inequality in (3.3) is, therefore, the more restrictive leading to the second inequality in (3.1). The inequalities in (3.2) are verified similarly.

The $\{1,2,3\}$ principal minor and the determinant must also be checked for positivity. We will first verify that $\operatorname{det}(\hat{A}[1,2,3])>0$. Note that the $\{1,2,3\}$ principal minor in the TN completion in $(2.3)$ is $\left(1-s_{1} t_{1}\right)\left(1-s_{2} t_{2}\right)$ and is positive since the two factors come from minors of fully specified 2-by-2 submatrices. Using Sylvester's determinantal inequality (which we use several times hereafter), this gives

$$
1>\frac{-s_{1} t_{1} s_{2} t_{2}}{1-s_{1} t_{1}-s_{2} t_{2}} .
$$

The $\{1,2,3\}$ principal minor of $\hat{A}$ is

$$
\operatorname{det}(\hat{A}[1,2,3])=1+t_{1} t_{2} s_{1} s_{2} \frac{1}{r}-s_{1} t_{1}-s_{2} t_{2}+s_{1} s_{2} \frac{s_{4} q}{t_{3} p}\left(1-\frac{1}{r}\right) .
$$


Since the last term is positive $(r>1)$ this minor is positive if

$$
1+t_{1} t_{2} s_{1} s_{2} \frac{1}{r}-s_{1} t_{1}-s_{2} t_{2}>0
$$

That is, if

$$
r>\frac{-s_{1} t_{1} s_{2} t_{2}}{1-s_{1} t_{1}-s_{2} t_{2}}
$$

which is the case by (3.4) and $r>1$.

In order for the determinant of $\hat{A}$ to be positive we must show (again by Sylvester)

$$
\begin{aligned}
\operatorname{det}(\hat{A})= & \frac{1}{1-s_{2} t_{2}}\left[\left(1-s_{1} t_{1}\right)\left(1-s_{2} t_{2}\right)-s_{1} s_{2}\left(1-\frac{1}{r}\right)\left(t_{1} t_{2}-\frac{s_{4}}{t_{3}} \frac{q}{p}\right)\right] \\
& *\left[\left(1-s_{2} t_{2}\right)\left(1-s_{3} t_{3}\right)-t_{2} t_{3}\left(1-\frac{1}{p}\right)\left(s_{2} s_{3}-\frac{t_{4}}{s_{1}} \frac{s}{r}\right)\right] \\
& -\left[\left(1-\frac{1}{p}\right)\left(t_{1} t_{2} t_{3}-s_{4} \frac{q}{p}\right)-s_{4}\left(\frac{q}{p^{2}}-1\right)\left(1-s_{2} t_{2}\right)\right] \\
& *\left[\left(1-\frac{1}{r}\right)\left(s_{1} s_{2} s_{3}-t_{4} \frac{s}{r}\right)-\left(\frac{s}{r^{2}}-1\right)\left(1-s_{2} t_{2}\right)\right]
\end{aligned}
$$

$>0$.

By $(3.1)$ and $(3.2), s, q \rightarrow 1$ as $r, p \rightarrow 1^{+}$and then $\operatorname{det}(\hat{A}) \rightarrow \operatorname{det}(\hat{B})=\left(1-s_{1} t_{1}\right)(1-$ $\left.s_{2} t_{2}\right)\left(1-s_{3} t_{3}\right)>0$. Therefore, $p, q, r, s$ can be chosen close enough to 1 , while still satisfying the constraints, so that $\hat{A}$ is a TP completion of $A$.

We now have a TP completion of the 4-by-4 partial TP matrix, the graph of whose specified entries is a cycle. This is the base case for the induction in the proof of the $n$-by- $n$ case. For an $n$-by- $n$ TP matrix $A$, it is easy to append a row (or column) $b^{T}$ to $A$ and get an $(n+1)$-by- $n$ TP matrix

$$
\hat{A}=\left[\begin{array}{c}
b^{T} \\
A
\end{array}\right] .
$$

For the induction step in the proof of the TP cycle completion in the general case we prepend the completed $(n-1)$-by- $(n-1)$ submatrix with a row along the top, but the first and last entries of that row must be specified. That $n$-by- $(n-1)$ matrix is then prepended with a column on the left to get a TP completion. The following lemma shows how to add a row to a TP matrix while maintaining the specified data in the first and last positions of the row.

LEMmA 3.2. Let the $n$-by-n matrix $A=\left(a_{i j}\right)$ be TP and let $b_{1} a_{1 n}>a_{11} b_{n}$. The partial TP matrix

$$
B=\left[\begin{array}{cccc}
b_{1} & ? & \cdots & \\
& A & & \\
& & &
\end{array}\right]
$$


has a TP completion.

Proof. Consider the matrix

$$
\left[\begin{array}{ccccc}
b_{1}^{\prime} & b_{2}^{\prime} & \cdots & b_{n-1}^{\prime} & b_{n} \\
& A &
\end{array}\right]
$$

in which the first row is proportional to the first row of $A$. The matrix in (3.5) is TN. Now, raise $b_{1}^{\prime}$ to $b_{1}$ to get the TN matrix

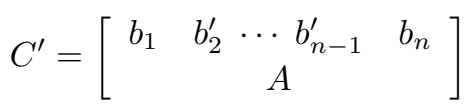

in which all minors involving the first row and column are positive (as are all minors not involving the first and second rows). Then $C^{\prime}$ is a TN completion of $B$.

Now consider the entries $b_{2}^{\prime}, \ldots, b_{n-1}^{\prime}$. Increasing $b_{2}^{\prime}$ will decrease minors that involve the first row and first and second columns of $C^{\prime}$, but these minors are all positive. On the other hand, increasing $b_{2}^{\prime}$ will increase minors that involve the first row and second column (but not the first column). These minors are all positive or 0 . So, the net effect of increasing $b_{2}^{\prime}$ is that some 0 minors will increase and some positive minors will decrease. Choose $\beta_{2}>b_{2}^{\prime}$ so that no positive minor becomes nonpositive.

The process can be continued by next considering $b_{3}^{\prime}$ followed by $b_{4}^{\prime}$, etc. At each step, the entry $b_{i}^{\prime}$ can be increased to $\beta_{i}, i=3, \ldots, n-1$ in such a way that no positive minor becomes nonpositive and all zero minors involving that entry become positive. This process results in the matrix

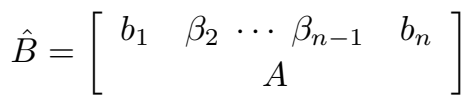

which is a TP completion of $B$.

Lemma 3.2 is used to prove the following:

Lemma 3.3. Let $A$ be an $(n-1)$-by- $(n-1)$ TP matrix. Then, the $n$-by-n partial TP matrix

$$
G=\left[\begin{array}{ccccc}
1 & b_{2} & ? & \cdots ? & b_{n} \\
c_{2} & & & \\
? & & & \\
\vdots & & A & \\
? & & & \\
c_{n} & & &
\end{array}\right]
$$

has a TP completion. 
Proof. Begin by completing the first row of

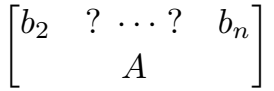

using the claim of Lemma 3.2 to obtain

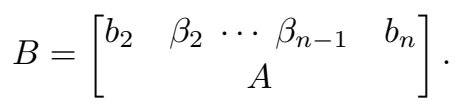

It is easily verified that

$$
C=\left[\begin{array}{cc}
1 & \\
c_{2} & \\
? & B \\
\vdots & \\
? & \\
c_{n} &
\end{array}\right]
$$

is partial TP.

A TP completion of $C$ can be found using the same bordering technique as in the proof of Lemma 3.2. Begin by bordering $B$ in the left side with a column that is a $\frac{c_{n}}{a_{n-1,1}}$ times the first column of $B$. By using this multiple we obtain $c_{n}$ in the $n, 1$ entry, resulting in the TN matrix

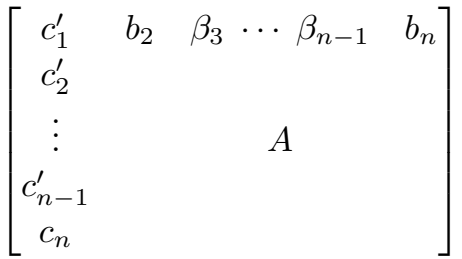

in which all minors involving the first and second columns are zero and minors not involving both columns are positive. The 1, 1 entry of the matrix in (3.6) is $c_{1}^{\prime}=\frac{b_{2} c_{n}}{a_{n-1,1}}$ which is less than 1 . Increasing this entry to 1 increases any minors involving the first row and column. Thus, all principal minors (including the determinant) are positive.

Next, we need to increase $c_{2}^{\prime}$ to $c_{2}$ while maintaining nonnegativity of all minors and ensuring that no positive minors become nonpositive. That is, we have to check that the matrix

$$
C^{\prime}=\left[\begin{array}{ccccc}
1 & b_{2} & \beta_{3} \cdots \beta_{n-1} & b_{n} \\
c_{2} & & & & \\
c_{3}^{\prime} & & & & \\
\vdots & & A & \\
c_{n-1}^{\prime} & & & \\
c_{n} & & & &
\end{array}\right]
$$


remains TN. We only need to check minors that include rows 1 and 2 and column 1 . The affected minors not containing row 1 will only increase since $c_{2}$ will be in the 1,1 position of the submatrix.

Let $x^{T}=\left(b_{2}, \beta_{3}, \ldots, \beta_{n-1}, b_{n}\right)$ and $y^{T}=\left(c_{2}, c_{3}^{\prime}, \ldots, c_{n-1}^{\prime}, c_{n}\right)$ and let $\sigma, \tau \subseteq N=$ $\{1,2, \ldots, n\}$ be such that $|\sigma|=|\tau|=k, 1,2 \in \sigma, 1 \in \tau$. If $W=C^{\prime}[\sigma \mid \tau]$, and if $x_{\tau}$, $y_{\sigma}$, and $H$ are the submatrices of $x, y$, and $A$, respectively, that are conformal with $C^{\prime}[\sigma \mid \tau]$ then

$$
W=\left[\begin{array}{cc}
1 & x_{\tau}^{T} \\
y_{\sigma} & H
\end{array}\right]
$$

Using the up-column elimination described in [7] (that preserves TN) to annihilate the entries below the 1,1 entry of $W$ we see that the determinant of $W$ is

$$
\operatorname{det} W=\operatorname{det}\left[\begin{array}{cc}
1 & x_{\tau}^{T} \\
0 & \\
\vdots & \tilde{H} \\
0 &
\end{array}\right]=\operatorname{det} \tilde{H}
$$

There are two cases to consider when finding $\operatorname{det} \tilde{H}$. If $\tau_{2}=2$, then, since the affected entries of the first column of $C^{\prime}$ are a multiple of the first column of $A$ the above elimination gives

$$
\tilde{H}=\left[\begin{array}{llll}
1 & a_{1, \tau_{3}}-c_{2} x_{\tau_{3}} & \cdots & a_{1, \tau_{k}}-c_{2} x_{\tau_{k}} \\
0 & & & \\
\vdots & & \tilde{H}(1) & \\
0 & &
\end{array}\right]
$$

and $\operatorname{det} W=\operatorname{det}(\tilde{H}(1))$ which equals the determinant of the corresponding submatrix of $A$. Since $A$ is TP, this determinant is positive.

The more subtle case is that in which $\tau_{2} \neq 2$. In this case, the submatrix $\tilde{H}$ has the form

$$
\tilde{H}=\left[\begin{array}{lll}
a_{1, \tau_{2}}-c_{2} x_{\tau_{2}} & \cdots & a_{1, \tau_{k}}-c_{2} x_{\tau_{k}}
\end{array}\right]
$$

in which $\tilde{H}_{1}$ is $\tilde{H}$ with the first row deleted. Then, by linearity of the determinant

$$
\operatorname{det} \tilde{H}=\operatorname{det} H-c_{2} \operatorname{det}\left[\begin{array}{lll}
x_{\tau_{2}} & \cdots & x_{\tau_{k}} \\
& \tilde{H}_{1} &
\end{array}\right]=\operatorname{det} H-c_{2} \operatorname{det} \tilde{H}_{x} \text {, }
$$

in which $\tilde{H}_{x}=\left[\begin{array}{lll}x_{\tau_{2}} & \cdots & x_{\tau_{k}} \\ & \tilde{H}_{1} & \end{array}\right]$. 
The determinant of $W$ is positive if $c_{2}<\frac{\operatorname{det} H}{\operatorname{det} \tilde{H}_{x}}$. From the original partial matrix we have that $1-b_{2} c_{2}>0$ so that $1 / b_{2}>c_{2}$. We will show that $1 / b_{2}<\frac{\operatorname{det} H}{\operatorname{det} \tilde{H}_{x}}$ and, thus $\operatorname{det} W>0$. If $\tau$ is changed to $\hat{\tau}$ so that $1 \notin \hat{\tau}$ and $\hat{\tau}_{1}=2$, then $C^{\prime}[\sigma \mid \hat{\tau}]$ is

$$
C^{\prime}[\sigma \mid \hat{\tau}]=\left[\begin{array}{cc}
b_{2} & x_{\hat{\tau}}^{T} \\
a_{\sigma_{2}, 1} & \\
\vdots & H \\
a_{\sigma_{k}, 1} &
\end{array}\right]
$$

which is a submatrix of the TP matrix $B$ and, therefore, has positive determinant. The same step of elimination that created zeros in the first column of $W$ also creates zeros in the first column of $C^{\prime}[\sigma \mid \hat{\tau}]$ below the 1,1 entry. The matrix $H$ is then reduced to

$$
\left[\begin{array}{ccc}
a_{1, \tau_{2}}-\frac{x_{\tau_{2}}}{b_{2}} & \ldots & a_{1, \tau_{k}}-\frac{x_{\tau_{k}}}{b_{2}} \\
& \tilde{H}_{1} &
\end{array}\right]
$$

and $0<\operatorname{det} C^{\prime}[\sigma \mid \hat{\tau}]=b_{2} \operatorname{det} \tilde{H}-\operatorname{det} \tilde{H}_{x}$ so that $1 / b_{2}<\frac{\operatorname{det} H}{\operatorname{det} \tilde{H}_{x}}$. Therefore, $C^{\prime}$ remains $\mathrm{TN}$ when $c_{2}^{\prime}$ is raised to $c_{2}$ (and keeps the determinant positive).

The same argument applied the $b_{j}$ 's in the proof of Lemma 3.2 can be applied to the $c_{i}$ 's in the first column of $C^{\prime}$ sequentially raising each $c_{i}$ to $\gamma_{i}$ resulting in a TP completion of the partial TP matrix $G$.

These TP lemmas allow us to state and prove the TP cycle completion result in the same manner as the proof in the TN case. The main result is:

TheOREM 3.4. Suppose that

$$
A=\left[\begin{array}{ccccc}
a_{11} & a_{12} & & & a_{1 n} \\
a_{21} & a_{22} & a_{23} & ? & \\
& a_{32} & \ddots & \ddots & \\
& ? & \ddots & \ddots & a_{n-1, n} \\
a_{n 1} & & & a_{n, n-1} & a_{n n}
\end{array}\right]
$$

is a partial TP matrix. Then $A$ has a TP completion if and only if:

$$
a_{1 n} a_{22} \cdots a_{n-1, n-1}<a_{12} a_{23} \cdots a_{n-1, n}
$$

and

$$
a_{n 1} a_{22} \cdots a_{n-1, n-1}<a_{21} a_{32} \cdots a_{n, n-1} .
$$


4. Additional comments. By a monotonically labelled block (MLB) graph $G$, we mean one that can be decomposed into $k$ induced subgraphs $G_{1}, G_{2}, \ldots, G_{k}$ in which the maximum label in $G_{i}$ is the minimum label of $G_{i+1}, i=1,2, \ldots, k-1$, the vertex set of $G_{i}$ intersects that of $G_{j}$ if and only if $j=i+1$ and then in exactly one vertex, and there are no edges between vertices of one subgraph and those of another. (Thus, the vertex of intersection of $G_{i}$ and $G_{i+1}$ is a cut vertex of $G$.) We note that a partial TN or TP matrix, the graph of whose specified entries is a MLB graph of $G$ has a TN completion, respectively a TP completion, if and only if the principal submatrix associated with each block $G_{i}$ has the appropriate completion (based upon the results of [8] in the TN case and [9] in the TP case). Now, if each block is either a clique or a properly labelled cycle, we have complete conditions for TN or TP completability.

\section{REFERENCES}

[1] W. Barrett, C.R. Johnson, and R. Loewy. The real positive definite completion problem: cycle completability. Mem. Amer. Math. Soc., 122 (584), 1996.

[2] W. Barrett, C.R. Johnson, and P. Tarazaga. The real positive definite completion problem for a simple cycle. Linear Algebra Appl., 192:3-31, 1993.

[3] E. Dryden and C.R. Johnson. Totally Nonnegative Completions. Research Experiences for Undergraduates Research Publications of the College of William and Mary, Summer 1997. Reported to NSF in Summer 1997 by C.R. Johnson, advising Emily Dryden. Supported by National Science Foundation grant DMS 96-19577.

[4] E. Dryden, C.R. Johnson, and B.K. Kroschel. Adjacent edge conditions for the totally nonnegative completion problem. Linear Multilinear Algebra, 56:261-277, 2008.

[5] S. Fallat and C.R. Johnson. Totally Nonnegative Matrices. Princeton University Press, Princeton, to appear.

[6] S. Fomin and A. Zelevinsky. Total positivity: Tests and parametrizations. Math. Intelligencer, 22:23-33, 2000.

[7] M. Gasca and J.M. Peña. On factorizations of totally positive matrices. Math. Appl., Kluwer Academic, Dordrecht, The Netherlands, 359:109-130, 1996.

[8] C.R. Johnson, B.K. Kroschel, and M. Lundquist. The totally nonnegative completion problem. Topics in Semidefinite and Interior-point Methods, Fields Inst. Commun., Amer. Math. Soc., Providence, 18:97-107, 1998.

[9] C.R. Johnson and C. Negron. Totally positive completions for monotonically labeled block clique graphs. Electron. J. Linear Algebra, 18:146-161, 2009.

[10] C. Jordan and J.R. Torregrosa. The totally positive completion problem. Linear Algebra Appl., 393:259-274, 2004. 